\title{
Recurrence equation and integral representation of Apéry sums
}

\author{
Michael Uhl ${ }^{1}$
}

Received: 9 October 2019 / Revised: 6 April 2020 / Accepted: 27 May 2020 / Published online: 30 June 2020

(c) The Author(s) 2020

\begin{abstract}
Various methods are used to investigate sums involving a reciprocal central binomial coefficient and a power term. In the first part, new functions are introduced for calculation of sums with a negative exponent in the power term. A recurrence equation for the functions provides an integral representation of the sums using polylogarithm functions. Thus polylogarithms and, in particular, zeta values can be expressed via these functions, too. In the second part, a straightforward recurrence formula is derived for sums having a positive exponent in the power term. Finally, two interesting cases of double sums are presented.
\end{abstract}

Keywords Apéry sum · Zeta function · Polylogarithm · Central binomial coefficient Mathematics Subject Classification 11M06 $11 \mathrm{G} 55 \cdot 11 \mathrm{~B} 65$

\section{Introduction and main results}

The subject of this article are infinite sums, $A_{m}^{+}$and $A_{m}^{-}$, involving a reciprocal central binomial coefficient $1 /\left(\begin{array}{c}2 k \\ k\end{array}\right)$ and a power term $k^{-m}$ :

$$
A_{m}^{+}=\sum_{k=1}^{\infty} \frac{1}{k^{m}\left(\begin{array}{c}
2 k \\
k
\end{array}\right)}, \quad A_{m}^{-}=\sum_{k=1}^{\infty} \frac{(-1)^{k-1}}{k^{m}\left(\begin{array}{c}
2 k \\
k
\end{array}\right)} .
$$

In the context of these sums-which we also call the Apéry sums-Roger Apéry proved the irrationality of $\pi^{2}$ and $\zeta(3)$ [1,9,13]. Furthermore, the tight relationship between these sums and values of the zeta function are the topic of many publications [2,6-8,10-14].

Michael Uhl

michael_uhl@t-online.de

1 Erbsengasse 1 F, 64404 Bickenbach, Germany 
Whereas there exist closed-form expressions for $A_{1}^{+}, A_{2}^{+}, A_{1}^{-}, A_{2}^{-}$, and as well $A_{4}^{+}$, see for instance [10-13], the sums $A_{m}^{+}$and $A_{m}^{-}$for $m>4$ can only be expressed by means of polylogarithmic ladders or multiple Clausen values [2,7]. In particular,

$$
\begin{aligned}
& A_{1}^{+}=\frac{\sqrt{3} \pi}{9}, \quad A_{2}^{+}=\frac{\pi^{2}}{18}=\frac{\zeta(2)}{3}, \quad A_{4}^{+}=\frac{17 \pi^{4}}{3240}=\frac{17 \zeta(4)}{36}, \\
& A_{1}^{-}=\frac{2 \ln \phi}{\sqrt{5}}, \quad A_{2}^{-}=2 \ln ^{2} \phi, \quad A_{3}^{-}=\frac{2 \zeta(3)}{5},
\end{aligned}
$$

where $\phi=(\sqrt{5}+1) / 2$, see e.g. $[2,11-13]$.

In this work two approaches are presented to calculate the sums with a negative exponent in the power term, $A_{m}^{+}$and $A_{m}^{-}$for $m>0$. First, using a reformulation of the reciprocal central binomial coefficient we define the functions $A_{m}^{+}(x)$ and $A_{m}^{-}(x)$ :

$$
A_{m}^{ \pm}(x)=\sum_{j=1}^{\infty} \frac{(-x)^{j}}{j} a_{m j}^{ \pm}, \quad a_{m j}^{ \pm}=\sum_{k=1}^{j-1}\left(\begin{array}{c}
k-1 \\
j-k-1
\end{array}\right) \frac{(\mp 1)^{k-1}}{k^{m-1}}
$$

These functions $A_{m}^{+}(x)$ and $A_{m}^{-}(x)$ together with their derivatives $A_{m}^{\prime+}(x)$ and $A_{m}^{\prime-}(x)$ relate the Apéry sums $A_{m}^{+}$and $A_{m}^{-}$with the polylogarithm functions $\operatorname{Li}_{n}(x)$, defined as

$$
\mathrm{Li}_{n}(x)=\sum_{k=1}^{\infty} \frac{x^{k}}{k^{n}}, \quad \operatorname{Li}_{n}^{\prime}(x)=\frac{1}{x} \operatorname{Li}_{n-1}(x)
$$

Theorem 1.1 $A_{m}^{+}=A_{m}^{+}(1), A_{m}^{-}=A_{m}^{-}(1)$.

Theorem 1.2

$$
A_{m}^{\prime+}(x)=\frac{\operatorname{Li}_{m-1}\left(x-x^{2}\right)}{1-x}, \quad A_{m}^{\prime-}(x)=\frac{\operatorname{Li}_{m-1}\left(x^{2}-x\right)}{x-1} .
$$

For the proofs of Theorems 1.1 and 1.2 see (11) and Lemma 2.1 in Sect. 2.

Thus, integrating (5) the Apéry sums for $m>0$ can be calculated as

$$
A_{m}^{+}=\int_{0}^{1} \frac{\operatorname{Li}_{m-1}\left(x-x^{2}\right)}{1-x} \mathrm{~d} x, \quad A_{m}^{-}=\int_{0}^{1} \frac{\operatorname{Li}_{m-1}\left(x^{2}-x\right)}{x-1} \mathrm{~d} x .
$$

Furthermore, as shown in Sect. 2, the polylogarithm $\mathrm{Li}_{m}(z)$, and in particular its value $\mathrm{Li}_{m}(1)=\zeta(m)$, can be expressed vice versa in terms of $A_{m}(x)$.

In Sect. 3 we derive an integral representation of the reciprocal central binomial coefficient, which gives us a direct integral formula of the sums, see (6). In Sect. 4 we calculate the integrals for $m=1,2,3$.

Due to the exponential convergence of the reciprocal binomial coefficients even the sums with a positive exponent in the power term, $A_{-m}^{+}$and $A_{-m}^{-}$for $m \geqslant 0$, converge. These sums up to $m=3$ are studied in various articles $[11,12,15]$. In this work a straightforward recurrence formula is presented to calculate them. 


\section{Theorem 1.3}

$$
\begin{aligned}
& 3 A_{-m}^{+}=1+2 A_{1-m}+\sum_{j=0}^{m-1}\left(\begin{array}{c}
m \\
j
\end{array}\right) A_{-j}, \\
& 5 A_{-m}^{-}=1+2 A_{1-m}-\sum_{j=0}^{m-1}\left(\begin{array}{c}
m \\
j
\end{array}\right) A_{-j} .
\end{aligned}
$$

For the proof see (26) in Sect. 5. By reiterative application of (7), the sums $A_{-m}^{+}$and $A_{-m}^{-}$are calculated as linear combinations of 1 and $A_{1}^{+}$, respectively 1 and $A_{1}^{-}$, where $A_{1}^{+}=\sqrt{3} \pi / 9$ and $A_{1}^{-}=2 \ln \phi / \sqrt{5}$, see (2).

Theorem 1.4 The numbers $3^{m} A_{-m}^{+}$and $5^{m} A_{-m}^{-}$are sums of an integer and odd-and therefore non-vanishing-multiple of $2 A_{1}^{+}$, respectively $2 A_{1}^{-}$.

For the proof see Lemma 5.1 in Sect. 5. Since $\pi$ and (due to the Gelfond-Schneider theorem [3-5]) $\ln \phi$ are transcendental, $A_{1}^{+}$and $A_{1}^{-}$are transcendental, too. Then, due to Theorem $1.4, A_{-m}^{+}$and $A_{-m}^{-}$for all $m \geqslant 0$ are transcendental numbers. For instance, this is different from $A_{3}^{-}$which is proven only to be irrational [1].

Although there is up to now-with the exception of $A_{4}^{+}$-no closed-form expression for $A_{m}^{+}$and $A_{m}^{-}$for all $m \geqslant 3$, the sums of $A_{m}^{+}-1 / 2$ and $A_{m}^{-}-1 / 2$ over $m$ are calculated as follows.

\section{Theorem 1.5}

$$
S^{+}=\sum_{m=1}^{\infty}\left(A_{m}^{+}-\frac{1}{2}\right)=\frac{1-A_{1}^{+}}{2}, \quad S^{-}=\sum_{m=1}^{\infty}\left(A_{m}^{-}-\frac{1}{2}\right)=\frac{1-3 A_{1}^{-}}{2} .
$$

For the proof see (30) in Sect. 6. Finally, in Sect. 6 the identities, $0=6 A_{0}^{+} A_{2}^{+}-$ $4 A_{1}^{+} A_{2}^{+}-3 A_{1}^{+} A_{1}^{+}$and $0=10 A_{0}^{-} A_{2}^{-}-4 A_{1}^{-} A_{2}^{-}-5 A_{1}^{-} A_{1}^{-}$, are presented as vanishing double sums over the products $1 /\left[\left(\begin{array}{c}2 k \\ k\end{array}\right)\left(\begin{array}{c}2 j \\ j\end{array}\right)\right]$.

\section{Recurrence equation}

We show below that non-alternating sums $A_{m}^{+}$and alternating sums $A_{m}^{-}$subordinate, in general, to the same equations, except for a sign. First, we simplify the notation: $A_{m}:=A_{m}^{ \pm}, a_{m}:=a_{m}^{ \pm}$, and $A_{m}(x):=A_{m}^{ \pm}(x)$. Reiterative application of the recurrence formula for reciprocal binomial coefficients,

$$
\left(\begin{array}{l}
n \\
m
\end{array}\right)^{-1}=\frac{m}{m-1}\left[\left(\begin{array}{c}
n-1 \\
m-1
\end{array}\right)^{-1}-\left(\begin{array}{c}
n-1 \\
m
\end{array}\right)^{-1}\right]
$$


to the reciprocal central binomial coefficient $1 /\left(\begin{array}{c}2 k \\ k\end{array}\right)$ gives

$$
\left(\begin{array}{c}
2 k \\
k
\end{array}\right)^{-1}=k \sum_{j=0}^{k-1}\left(\begin{array}{c}
k-1 \\
j
\end{array}\right) \frac{(-1)^{j}}{k+j+1}=k \sum_{j=k+1}^{2 k}\left(\begin{array}{c}
k-1 \\
j-k-1
\end{array}\right) \frac{(-1)^{j-k-1}}{j} .
$$

Thus the sums $A_{m},(1)$, for $m \geqslant 1$, can be reformulated via coefficients $\alpha_{m j k}$ as

$$
\begin{aligned}
A_{m} & =\sum_{k=1}^{\infty} \frac{( \pm 1)^{k-1}}{k^{m}\left(\begin{array}{c}
2 k \\
k
\end{array}\right)}=\sum_{k=1}^{\infty} \sum_{j=k+1}^{2 k} \frac{(-1)^{j}}{j} \alpha_{m j k}, \\
\alpha_{m j k} & =\left(\begin{array}{c}
k-1 \\
j-k-1
\end{array}\right) \frac{(\mp 1)^{k-1}}{k^{m-1}} .
\end{aligned}
$$

Now, for a more detailed investigation of the sums $A_{m}$ the functions $A_{m}(x)$ are introduced, see (3):

$$
\begin{aligned}
A_{m}(x) & =\sum_{j=1}^{\infty} \sum_{k=1}^{j-1} \frac{(-x)^{j}}{j} \alpha_{m j k}=\sum_{j=1}^{\infty} \frac{(-x)^{j}}{j} a_{m j}, \\
a_{m j} & =\sum_{k=1}^{j-1} \alpha_{m j k}=\sum_{k=1}^{j-1}\left(\begin{array}{c}
k-1 \\
j-k-1
\end{array}\right) \frac{(\mp 1)^{k-1}}{k^{m-1}} .
\end{aligned}
$$

In the end of this section the coefficients $a_{1 j}^{+}$and $a_{1 j}^{-}$are calculated. Convergence of the sums $A_{m}^{+}(x)$ and $A_{m}^{-}(x)$ is guaranteed for $|x|<1$, respectively $|x|<1 / \phi$, only. However, by the process of analytic continuation the order of summation in (10) can be switched and a comparison with (9) shows

$$
A_{m}(x)=\sum_{j=1}^{\infty} \sum_{k=1}^{j-1} \frac{(-x)^{j}}{j} \alpha_{m j k}=\sum_{k=1}^{\infty} \sum_{j=k+1}^{2 k} \frac{(-x)^{j}}{j} \alpha_{m j k}, \quad A_{m}(1)=A_{m} .
$$

The derivatives $A_{m}^{\prime}(x)$ and $A_{m}^{\prime \prime}(x)$ are given by

$$
A_{m}^{\prime}(x)=-\sum_{j=1}^{\infty}(-x)^{j-1} a_{m j}, \quad A_{m}^{\prime \prime}(x)=\sum_{j=1}^{\infty}(j-1)(-x)^{j-2} a_{m j} .
$$


For $m=1$ the derivative $A_{1}^{\prime}(x)$-as the analytic continuation of (12) — is calculated as

$$
\begin{aligned}
A_{1}^{\prime}(x) & =-\sum_{j=1}^{\infty}(-x)^{j-1} \sum_{k=1}^{j-1}(\mp 1)^{k-1}\left(\begin{array}{c}
k-1 \\
j-k-1
\end{array}\right) \\
& =-\sum_{k=1}^{\infty} \sum_{j=1}^{k}(\mp 1)^{k-1}(-x)^{k+j-1}\left(\begin{array}{c}
k-1 \\
j-1
\end{array}\right) \\
& =x \sum_{k=1}^{\infty}( \pm x)^{k-1}(1-x)^{k-1}=\frac{x}{1 \pm\left(x^{2}-x\right)} .
\end{aligned}
$$

In fact, the coefficients $a_{m j}$ from (10) satisfy the identity

$$
\begin{aligned}
& (j-1) a_{m j}-a_{m-1, j}+(j-1) a_{m, j-1}-2 a_{m-1, j-1} \\
& \quad=\sum_{k=1}^{j-1} \frac{(\mp 1)^{k-1}}{k^{m-1}}\left((j-1-k)\left(\begin{array}{c}
k-1 \\
j-k-1
\end{array}\right)+(j-1-2 k)\left(\begin{array}{c}
k-1 \\
j-k-2
\end{array}\right)\right)=0 .
\end{aligned}
$$

Thus, for the functions $A_{m}(x)$ from (10) the following differential equation is obtained:

$$
\begin{aligned}
& \left(x^{2}-x\right) A_{m}^{\prime \prime}(x)+x A_{m}^{\prime}(x)-(2 x-1) A_{m-1}^{\prime}(x) \\
& =\sum_{j=1}^{\infty}(j-1) a_{m j}\left(x^{2}-x\right)(-x)^{j-2}-\sum_{j=1}^{\infty}\left[a_{m j} x-a_{m-1, j}(2 x-1)\right](-x)^{j-1} \\
& =\sum_{j=1}^{\infty}\left[(j-1) a_{m j}-a_{m-1, j}\right](-x)^{j-1}+\sum_{j=1}^{\infty}\left[j a_{m j}-2 a_{m-1, j}\right](-x)^{j} \\
& =\sum_{j=1}^{\infty}\left[(j-1) a_{m j}-a_{m-1, j}+(j-1) a_{m, j-1}-2 a_{m-1, j-1}\right](-x)^{j-1}=0 .
\end{aligned}
$$

Therefore, the derivatives $A_{m}^{\prime}(x)$ can be calculated recurrently:

$$
\frac{2 x-1}{x} A_{m-1}^{\prime}(x)=(x-1) A_{m}^{\prime \prime}(x)+A_{m}^{\prime}(x)=\left[(x-1) A_{m}^{\prime}(x)\right]^{\prime} .
$$

Since $A_{m-1}^{\prime}(0)=a_{m 1}=0$, by integration $A_{m}^{\prime}(x)$ can be written as

$$
A_{m}^{\prime}(x)=\frac{1}{x-1} \int_{0}^{x} \frac{2 t-1}{t} A_{m-1}^{\prime}(t) \mathrm{d} t .
$$


Lemma 2.1 The function $(x-1) A_{m}^{\prime}(x)$ and the polylogarithm $\mp \operatorname{Li}_{m-1}\left(\mp\left(x^{2}-x\right)\right)$ are identical:

$$
(x-1) A_{m}^{\prime}(x)=\mp \operatorname{Li}_{m-1}\left(\mp\left(x^{2}-x\right)\right) .
$$

Proof (i) For $m=1$ both sides are identical and (ii) for $m>1$ they are described by the same recurrence equation.

(i) In the case $m=1$, it follows from (13) and the definition $\operatorname{Li}_{0}(z)=z /(1-z)$ :

$$
(x-1) A_{1}^{\prime}(x)=\mp \operatorname{Li}_{0}\left(\mp\left(x^{2}-x\right)\right)=\frac{x^{2}-x}{1 \pm\left(x^{2}-x\right)} .
$$

(ii) Applying (15) to the recurrence equation of polylogarithm, $\operatorname{Li}_{m-1}^{\prime}(z)=$ $\mathrm{Li}_{m-2}(z) / z$ with $z=\mp\left(x^{2}-x\right)$, results in (14), again:

$$
\begin{aligned}
{\left[\mp \operatorname{Li}_{m-1}\left(\mp\left(x^{2}-x\right)\right)\right]^{\prime} } & =\mp(2 x-1) \frac{\operatorname{Li}_{m-2}\left(\mp\left(x^{2}-x\right)\right)}{x^{2}-x} \\
& =\left[(x-1) A_{m}^{\prime}(x)\right]^{\prime}=\frac{2 x-1}{x} A_{m-1}^{\prime}(x) .
\end{aligned}
$$

Due to the symmetry of (15),

$$
x A_{m}^{\prime}(1-x)=(1-x) A_{m}^{\prime}(x)
$$

and the transformation, $x \mapsto \frac{1}{2}-x$, the Apéry sums $A_{m}=A_{m}(1)$ are calculated as

$$
A_{m}=\int_{0}^{1} A_{m}^{\prime}(x) \mathrm{d} x=\int_{0}^{1} \frac{\operatorname{Li}_{m-1}\left(\mp\left(x^{2}-x\right)\right)}{\mp(x-1)} \mathrm{d} x=\int_{0}^{\frac{1}{2}} \frac{\operatorname{Li}_{m-1}\left( \pm\left(\frac{1}{4}-x^{2}\right)\right)}{ \pm\left(\frac{1}{4}-x^{2}\right)} \mathrm{d} x
$$

Integrating (16), the polylogarithm $\mp \mathrm{Li}_{m}\left(\mp\left(x-x^{2}\right)\right)$ can be described by the function $A_{m}(x)$ :

$$
\begin{aligned}
\mp \operatorname{Li}_{m}\left(\mp\left(x^{2}-x\right)\right) & =\int_{0}^{x} \frac{2 t-1}{t} A_{m}^{\prime}(t) \mathrm{d} t \\
& =\int_{0}^{x}\left(A_{m}^{\prime}(t)-A_{m}^{\prime}(1-t)\right) \mathrm{d} t=A_{m}(x)+A_{m}(1-x)-A_{m}(1)
\end{aligned}
$$

in which $\operatorname{Li}_{m}(0)=A_{m}(0)=0$ and the symmetry of (17) are used.

The values of the zeta function are given by $\zeta(m)=\mathrm{Li}_{m}(1)$. Using the definition of the coefficients $a_{m j}^{+}$from (9), and writing $\varphi=\exp (i \pi / 3)$ with $\varphi-\varphi^{2}=1$ and $1-\varphi=\varphi^{-1}$, one obtains 


$$
\begin{aligned}
\zeta(m) & =\operatorname{Li}_{m}(1)=\operatorname{Li}_{m}\left(\varphi-\varphi^{2}\right)=A_{m}^{+}(1)-A_{m}^{+}(\varphi)-A_{m}^{+}\left(\varphi^{-1}\right) \\
& =\sum_{j=1}^{\infty} \frac{(-1)^{j}}{j} a_{m j}^{+}\left(1-\varphi^{j}-\varphi^{-j}\right)=\sum_{j=1}^{\infty} \frac{(-1)^{j}}{j} a_{m j}^{+}\left(1-2 \cos \frac{j \pi}{3}\right) .
\end{aligned}
$$

Thus, the functions $A_{m}^{+}(x)$, or respectively the coefficients $a_{m j}^{+}$, enable a formulation for both the sums $A_{m}^{+}$and the values $\zeta(m)$.

Next, we want to focus on the coefficients $a_{m j}$ from (9) in the case $m=1$. Here, the coefficients $a_{1 j}^{+}$of the non-alternating sum $A_{1}^{+}(x)$ fulfil the equation

$$
a_{1 j}^{+}=\sum_{k=1}^{j-1}(-1)^{k-1}\left(\begin{array}{c}
k-1 \\
j-k-1
\end{array}\right)=-a_{1, j-1}^{+}-a_{1, j-2}^{+} .
$$

With $a_{11}^{+}=0$ and $a_{12}^{+}=1$ one gets recurrently,

$$
a_{1,3 j+1}^{+}=0, \quad a_{1,3 j+2}^{+}=1, \quad a_{1,3 j+3}^{+}=-1, \quad j \geqslant 0 .
$$

Hence, comparing the sum $A_{1}^{+}=A_{1}^{+}$(1) from (10) with (2) gives

$$
A_{1}^{+}=\sum_{j=1}^{\infty} \frac{(-1)^{j} a_{1 j}^{+}}{j}=\sum_{j=0}^{\infty} \frac{(-1)^{j}(6 j+5)}{(3 j+2)(3 j+3)}=\frac{\pi \sqrt{3}}{9}
$$

The coefficients $a_{1 j}^{-}$of the alternating sum $A_{1}^{-}(x)$ can be written as

$$
a_{1 j}^{-}=\sum_{k=1}^{j-1}\left(\begin{array}{c}
k-1 \\
j-k-1
\end{array}\right)=a_{1, j-1}^{-}+a_{1, j-2}^{-}
$$

With $a_{11}^{-}=0$ and $a_{12}^{-}=1$ the coefficients $a_{1, j+1}^{-}$are given by the Fibonacci numbers $F_{j}$ :

$$
a_{1, j+1}^{-}=F_{j}=\frac{\phi^{j}-(1-\phi)^{j}}{\sqrt{5}}, \quad j \geqslant 0, \quad \phi=\frac{\sqrt{5}+1}{2} .
$$

Since Fibonacci numbers grow exponentially with $\lim _{j \rightarrow \infty} F_{j} / F_{j-1}=\phi$, at $x=1$ the sum $A_{1}^{-}(1)=\sum_{j=1}^{\infty}(-1)^{j} a_{1 j}^{-} / j$ does not converge.

Having $\left|a_{1 j}^{+}\right| \leqslant 1$ for all $j$, the convergence radius of $A_{1}^{+}(x),(10)$, is $R_{1}^{+}=1$. The convergence radius of $A_{1}^{-}(x)$ is given by $R_{1}^{-}=\lim _{j \rightarrow \infty}\left|a_{1, j-1}^{-} / a_{1 j}^{-}\right|=1 / \phi$. Furthermore, with $\left|a_{m j}^{+}\right| \leqslant\left|a_{m-1, j}^{+}\right|$and $\left|a_{m j}^{-}\right| \leqslant\left|a_{m-1, j}^{-}\right|$it follows $R_{m}^{+} \geqslant 1$ and $R_{m}^{-} \geqslant 1 / \phi$. 


\section{Integral representation}

The integrals $A_{m}$ can be obtained directly by the integral representation of the reciprocal central binomial coefficients $1 /\left(\begin{array}{c}2 k \\ k\end{array}\right)$, see e.g. [15]. Looking at the integrals $\int_{0}^{1 / 2}\left(\frac{1}{4}-x^{2}\right)^{k-1} \mathrm{~d} x$, partial integration results in

$$
\int_{0}^{\frac{1}{2}}\left(\frac{1}{4}-x^{2}\right)^{k-1} \mathrm{~d} x=\frac{4 k+2}{k} \int_{0}^{\frac{1}{2}}\left(\frac{1}{4}-x^{2}\right)^{k} \mathrm{~d} x
$$

Thus, starting with $\int_{0}^{1 / 2}\left(\frac{1}{4}-x^{2}\right)^{0} \mathrm{~d} x=\frac{1}{2}$, we arrive to the integral by induction:

$$
\int_{0}^{\frac{1}{2}}\left(\frac{1}{4}-x^{2}\right)^{k} \mathrm{~d} x=\frac{k !(k+1) !}{(2 k+2) !}
$$

So, the reciprocal central binomial coefficients $1 /\left(\begin{array}{c}2 k \\ k\end{array}\right)$ are

$$
\left(\begin{array}{c}
2 k \\
k
\end{array}\right)^{-1}=\frac{k !^{2}}{(2 k) !}=k \int_{0}^{\frac{1}{2}}\left(\frac{1}{4}-x^{2}\right)^{k-1} \mathrm{~d} x .
$$

With (8) and the definition of the polylogarithm function, $\operatorname{Li}_{m}(z)=\sum_{k=1}^{\infty} k^{-m} z^{k}$, the sums $A_{m}$ can be calculated as

$$
A_{m}=\sum_{k=1}^{\infty} \frac{( \pm 1)^{k-1}}{k^{m}\left(\begin{array}{c}
2 k \\
k
\end{array}\right)}=\int_{0}^{\frac{1}{2}} \sum_{k=1}^{\infty} \frac{\left( \pm\left(\frac{1}{4}-x^{2}\right)\right)^{k-1}}{k^{m-1}} \mathrm{~d} x=\int_{0}^{\frac{1}{2}} \frac{\mathrm{Li}_{m-1}\left( \pm\left(\frac{1}{4}-x^{2}\right)\right)}{ \pm\left(\frac{1}{4}-x^{2}\right)} \mathrm{d} x
$$

in agreement with (18). A similar result was derived by Taylor [15].

Furthermore, calculating the integral formula for the coefficients $1 /\left(\begin{array}{c}2 k \\ k\end{array}\right)$ in (20) one obtains the identity

$$
\left(\begin{array}{c}
2 k \\
k
\end{array}\right)^{-1}=k \sum_{h=0}^{k-1}\left(\begin{array}{c}
k-1 \\
h
\end{array}\right) \int_{0}^{\frac{1}{2}} \frac{\left(-x^{2}\right)^{h}}{4^{k-1-h}} \mathrm{~d} x=\frac{2 k}{4^{k}} \sum_{h=0}^{k-1}\left(\begin{array}{c}
k-1 \\
h
\end{array}\right) \frac{(-1)^{h}}{2 h+1} .
$$

\section{Integrals for $A_{1}, A_{2}$, and $A_{3}$}

The calculation of the sums $A_{m}$ for $m>0$, and in particular $A_{1}$ and $A_{2}$, see (2), has been considered in many publications, see e.g. [11-13]. In this section we employ the integrals from (18) to calculate $A_{1}$ and $A_{2}$ and to give an integral formula of $A_{3}$. For $m=1$ the integral $A_{1}$ from (18) is given by $\operatorname{Li}_{0}(z)=z /(1-z)$ :

$$
A_{1}=\int_{0}^{\frac{1}{2}} \frac{\operatorname{Li}_{0}\left( \pm\left(\frac{1}{4}-x^{2}\right)\right)}{ \pm\left(\frac{1}{4}-x^{2}\right)} \mathrm{d} x=\int_{0}^{\frac{1}{2}} \frac{\mathrm{d} x}{1 \pm\left(x^{2}-\frac{1}{4}\right)}
$$


In particular, $A_{1}^{+}$and $A_{1}^{-}$are calculated as

$$
\begin{aligned}
& A_{1}^{+}=\int_{0}^{\frac{1}{2}} \frac{\mathrm{d} x}{\frac{3}{4}+x^{2}}=\left[\frac{2}{\sqrt{3}} \arctan \frac{2 x}{\sqrt{3}}\right]_{0}^{\frac{1}{2}}=\frac{2}{\sqrt{3}} \frac{\pi}{6}=\frac{\pi \sqrt{3}}{9}, \\
& A_{1}^{-}=\int_{0}^{\frac{1}{2}} \frac{\mathrm{d} x}{\frac{5}{4}-x^{2}}=\left[\frac{1}{\sqrt{5}} \ln \frac{\sqrt{5}+2 x}{\sqrt{5}-2 x}\right]_{0}^{\frac{1}{2}}=\frac{2}{\sqrt{5}} \ln \frac{\sqrt{5}+1}{2} .
\end{aligned}
$$

For $m=2$ the integral $A_{2}$ from (18) can be calculated via $\operatorname{Li}_{1}(z)=-\ln (1-z)$ :

$$
A_{2}= \pm \int_{0}^{1} \frac{\operatorname{Li}_{1}\left(\mp\left(x^{2}-x\right)\right)}{x} \mathrm{~d} x=\mp \int_{0}^{1} \frac{\ln \left(1 \pm\left(x^{2}-x\right)\right)}{x} \mathrm{~d} x \text {. }
$$

Having the factorizations,

$$
\begin{aligned}
& 1-x+x^{2}=(\varphi-x)\left(\varphi^{-1}-x\right), \quad \varphi=e^{i \pi / 3}, \varphi^{-1}=e^{-i \pi / 3}, \varphi+\varphi^{-1}=1 \\
& 1+x-x^{2}=(\phi-x)\left(\phi^{-1}+x\right), \quad \phi=\frac{\sqrt{5}+1}{2}, \phi^{-1}=\frac{\sqrt{5}-1}{2}, \phi-\phi^{-1}=1
\end{aligned}
$$

the integral $\int \ln (a \pm x) / x \mathrm{~d} x=\ln x \ln a-\operatorname{Li}_{2}(\mp x / a)$, the dilogarithm $\operatorname{Li}_{2}(0)=0$, and the Landen identities,

$$
\begin{aligned}
\operatorname{Li}_{2}\left(\varphi^{-1}\right)+\operatorname{Li}_{2}(\varphi) & =\operatorname{Li}_{2}(1-\varphi)+\operatorname{Li}_{2}\left(1-\varphi^{-1}\right)=-\frac{\ln ^{2} \varphi}{2}=\frac{\pi^{2}}{18} \\
\operatorname{Li}_{2}\left(\phi^{-1}\right)+\operatorname{Li}_{2}(-\phi) & =\operatorname{Li}_{2}\left(1-\phi^{-2}\right)+\operatorname{Li}_{2}\left(1-\phi^{2}\right)=-\frac{\ln ^{2} \phi^{2}}{2}=-2 \ln ^{2} \phi
\end{aligned}
$$

$A_{2}^{+}$and $A_{2}^{-}$are obtained as

$$
\begin{gathered}
A_{2}^{+}=-\int_{0}^{1} \frac{\ln \left(1-x+x^{2}\right)}{x} \mathrm{~d} x=\left[\operatorname{Li}_{2} \frac{x}{\varphi}+\operatorname{Li}_{2} \frac{x}{\varphi^{-1}}\right]_{0}^{1}=\frac{\pi^{2}}{18}, \\
A_{2}^{-}=\int_{0}^{1} \frac{\ln \left(1+x-x^{2}\right)}{x} \mathrm{~d} x=\left[-\operatorname{Li}_{2} \frac{x}{\phi}-\operatorname{Li}_{2} \frac{-x}{\phi^{-1}}\right]_{0}^{1}=2 \ln ^{2} \phi .
\end{gathered}
$$

The integral $A_{3}$ from (18) is calculated by integration of the dilogarithm $\mathrm{Li}_{2}$. Integrating by parts one finds

$$
A_{3}=\int_{0}^{\frac{1}{2}} \frac{\operatorname{Li}_{2}\left( \pm\left(\frac{1}{4}-x^{2}\right)\right)}{ \pm\left(\frac{1}{4}-x^{2}\right)} \mathrm{d} x=\int_{0}^{\frac{1}{2}} \frac{ \pm 2 x \ln \left(1 \pm\left(x^{2}-\frac{1}{4}\right)\right)}{x^{2}-\frac{1}{4}} \ln \frac{1+2 x}{1-2 x} \mathrm{~d} x
$$


Thus the sums $A_{3}^{+}$and $A_{3}^{-}=\frac{2}{5} \zeta(3)$, see (2), are given by the following integrals:

$$
\begin{aligned}
& A_{3}^{+}=\int_{0}^{\frac{1}{2}} \frac{\operatorname{Li}_{2}\left(\frac{1}{4}-x^{2}\right)}{\frac{1}{4}-x^{2}} \mathrm{~d} x=\int_{0}^{\frac{1}{2}} \frac{2 x \ln \left(\frac{3}{4}+x^{2}\right)}{x^{2}-\frac{1}{4}} \ln \frac{1+2 x}{1-2 x} \mathrm{~d} x \\
& A_{3}^{-}=\int_{0}^{\frac{1}{2}} \frac{\operatorname{Li}_{2}\left(x^{2}-\frac{1}{4}\right)}{x^{2}-\frac{1}{4}} \mathrm{~d} x=\int_{0}^{\frac{1}{2}} \frac{2 x \ln \left(\frac{5}{4}-x^{2}\right)}{\frac{1}{4}-x^{2}} \ln \frac{1+2 x}{1-2 x} \mathrm{~d} x=\frac{2 \zeta(3)}{5},
\end{aligned}
$$

leading to another integral form for $\zeta(3)$.

\section{Apéry sums for positive powers}

The sums for positive powers, $A_{-m}$ for $m \geqslant 0$, have also been studied in $[11,12,15]$. Calculation of $A_{0}, A_{-1}, A_{-2}$, and $A_{-3}$ was done by Lehmer [12]. In this section, a general recurrence formula for $A_{-m}$ is derived. The sums $A_{-m}$ converge, since $1 /\left(\begin{array}{c}2 k \\ k\end{array}\right)<\frac{k}{4^{k}}$ and

$$
\left|A_{-m}\right|=\left|\sum_{k=1}^{\infty} \frac{( \pm 1)^{k} k^{m}}{\left(\begin{array}{c}
2 k \\
k
\end{array}\right)}\right|<\sum_{k=1}^{\infty} \frac{k^{m+1}}{4^{k}}=\mathrm{Li}_{-(m+1)}\left(\frac{1}{4}\right)
$$

With (1) the expression $4 A_{m}-2 A_{m+1}$ can be calculated by

$$
\begin{aligned}
4 A_{m}-2 A_{m+1} & =\sum_{k=1}^{\infty} \frac{(4 k-2)( \pm 1)^{k-1}}{k^{m+1}\left(\begin{array}{c}
2 k \\
k
\end{array}\right)} \\
& =\sum_{k=1}^{\infty} \frac{( \pm 1)^{k-1}}{k^{m}\left(\begin{array}{c}
2 k-2 \\
k-1
\end{array}\right)}=1 \pm \sum_{k=1}^{\infty} \frac{( \pm 1)^{k-1}}{(k+1)^{m}\left(\begin{array}{c}
2 k \\
k
\end{array}\right)}
\end{aligned}
$$

Thus, we obtain in the case of positive powers, with $m \geqslant 0$, that

$$
4 A_{-m}-2 A_{1-m}=1 \pm \sum_{k=1}^{\infty}(k+1)^{m} \frac{( \pm 1)^{k-1}}{\left(\begin{array}{c}
2 k \\
k
\end{array}\right)}=1 \pm \sum_{j=0}^{m}\left(\begin{array}{c}
m \\
j
\end{array}\right) A_{-j}
$$

This directly leads to the recurrence equation for the sums $A_{-m}$ :

$$
A_{0}=\frac{1+2 A_{1}}{4 \mp 1}, \quad A_{-m}=\frac{1}{4 \mp 1}\left(1+2 A_{1-m} \pm \sum_{j=0}^{m-1}\left(\begin{array}{c}
m \\
j
\end{array}\right) A_{-j}\right)
$$


Here, the sums $A_{-m}$ can be calculated recurrently starting with $A_{1}$. For $0 \leqslant m \leqslant 4$ the sums $A_{-m}^{+}$and $A_{-m}^{-}$are given by:

$$
\begin{aligned}
& A_{1}^{+}=\frac{\pi \sqrt{3}}{9}, \\
& A_{0}^{+}=\frac{1+2 A_{1}^{+}}{3}=\frac{1}{3}+\frac{2 A_{1}^{+}}{3}, \\
& A_{-1}^{+}=\frac{1+3 A_{0}^{+}}{3}=\frac{2}{3}+\frac{2 A_{1}^{+}}{3}, \\
& A_{-2}^{+}=\frac{1+4 A_{-1}^{+}+1 A_{0}^{+}}{3}=\frac{4}{3}+\frac{10 A_{1}^{+}}{9}, \\
& A_{-3}^{+}=\frac{1+5 A_{-2}^{+}+3 A_{-1}^{+}+1 A_{0}^{+}}{3}=\frac{10}{3}+\frac{74 A_{1}^{+}}{27}, \\
& A_{-4}^{+}=\frac{1+6 A_{-3}^{+}+6 A_{-2}^{+}+4 A_{-1}^{+}+1 A_{0}^{+}}{3}=\frac{32}{3}+\frac{238 A_{1}^{+}}{27} \\
& A_{1}^{-}=\frac{2 \ln \phi}{\sqrt{5}}, \\
& A_{0}^{-}=\frac{1+2 A_{1}^{-}}{5}=\frac{1}{5}+\frac{2 A_{1}^{-}}{5}, \\
& A_{-3}^{-}=\frac{1-1 A_{-2}^{-}-3 A_{-1}^{-}-1 A_{0}^{-}}{5}=-\frac{2}{125}-\frac{14 A_{1}^{-}}{125}, \\
& A_{-1}^{-}=\frac{1+1 A_{0}^{-}}{5}=\frac{6}{25}+\frac{2 A_{1}^{-}}{25}, \\
& A_{-2}^{-}=\frac{1+0 A_{-1}^{-}-1 A_{0}^{-}}{5}=\frac{4}{25}-\frac{2 A_{1}^{-}}{25}, \\
&-325
\end{aligned}
$$

Using the substitution $\widetilde{A}_{-m}=(4 \mp 1)^{m+1} A_{-m}$, (26) gives

$$
\begin{aligned}
\widetilde{A}_{0} & =1+2 A_{1}, \quad \widetilde{A}_{-m}=(4 \mp 1)^{m}+\sum_{j=0}^{m-1} w_{m j} \widetilde{A}_{-j}, \\
w_{m j} & =2 \delta_{m-1, j} \pm\left(\begin{array}{c}
m \\
j
\end{array}\right)(4 \mp 1)^{m-1-j} .
\end{aligned}
$$

Here, $w_{m j}$ are the weights of $\widetilde{A}_{-j}$ contributing to $\widetilde{A}_{-m}$.

Lemma 5.1 The numbers $\widetilde{A}_{-m}$ are sums of an integer and an odd multiple of $2 A_{1}$. 
Proof First we prove $\widetilde{A}_{0}$ is a sum of an integer and an odd multiple of $2 A_{1}$. All weights $w_{m j}$ are integer numbers and the sum of weights,

$$
\sum_{j=0}^{m-1} w_{m j}=2 \pm \sum_{j=0}^{m-1}(4 \mp 1)^{m-1-j}\left(\begin{array}{c}
m \\
j
\end{array}\right)=2 \pm \frac{(5 \mp 1)^{m}-1}{4 \mp 1}
$$

is an odd number. Then, by induction, each number $\widetilde{A}_{-m}$ is a sum of an integer and an odd multiple of $2 A_{1}$.

\section{Further relations on Apéry sums}

In the same way as the zeta function $\zeta(m)=\sum_{k=1}^{\infty} k^{-m}$ and eta function $\eta(m)=$ $\sum_{k=1}^{\infty}(-1)^{k-1} k^{-m}$ converge to 1 for $m \rightarrow \infty$, the sums $A_{m}=\sum_{k=1}^{\infty}( \pm 1)^{k-1} k^{-m} /$ $\left(\begin{array}{c}2 k \\ k\end{array}\right)$ converge to $\frac{1}{2}$, since all terms for $k \geqslant 2$ vanish when $m \rightarrow \infty$, and the term for $k=1$ establishes the limit. The sum of Apéry sums, $S=\sum_{m=1}^{\infty}\left(A_{m}-\frac{1}{2}\right)$, can be written as

$$
S=\sum_{m=1}^{\infty}\left(A_{m}-\frac{1}{2}\right)=\sum_{m=1}^{\infty} \sum_{k=2}^{\infty} \frac{( \pm 1)^{k-1}}{k^{m}\left(\begin{array}{c}
2 k \\
k
\end{array}\right)}=\sum_{k=2}^{\infty} \frac{( \pm 1)^{k-1}}{(k-1)\left(\begin{array}{c}
2 k \\
k
\end{array}\right)}
$$

To calculate $S$, first we construct the sum of $4 A_{m}-2 A_{m+1}-1$, (25), over $m$ :

$$
\begin{aligned}
\sum_{m=1}^{\infty}\left(4 A_{m}-2 A_{m+1}-1\right) & = \pm \sum_{m=1}^{\infty} \sum_{k=1}^{\infty} \frac{( \pm 1)^{k-1}}{(k+1)^{m}\left(\begin{array}{c}
2 k \\
k
\end{array}\right)} \\
& = \pm \sum_{k=1}^{\infty} \frac{( \pm 1)^{k-1}}{k\left(\begin{array}{c}
2 k \\
k
\end{array}\right)}= \pm A_{1}
\end{aligned}
$$

Regrouping (29) leads to

$$
\sum_{m=1}^{\infty}\left(4 A_{m}-2 A_{m+1}-1\right)=4 \sum_{m=1}^{\infty}\left(A_{m}-\frac{1}{2}\right)-2 \sum_{m=2}^{\infty}\left(A_{m}-\frac{1}{2}\right)=2 S+2 A_{1}-1
$$

Comparing one gets

$$
2 S+(2 \mp 1) A_{1}-1=0 .
$$


Thus, the sum $S^{+}$of the non-alternating sums $A_{m}^{+}$and the sum $S^{-}$of the alternating sums $A_{m}^{-}$are given by

$$
\begin{aligned}
& S^{+}=\sum_{m=1}^{\infty}\left(A_{m}^{+}-\frac{1}{2}\right)=\sum_{k=2}^{\infty} \frac{1}{(k-1)\left(\begin{array}{c}
2 k \\
k
\end{array}\right)}=\frac{1}{2}-\frac{1}{2} A_{1}^{+}=\frac{1}{2}-\frac{\pi \sqrt{3}}{18} \\
& S^{-}=\sum_{m=1}^{\infty}\left(A_{m}^{-}-\frac{1}{2}\right)=\sum_{k=2}^{\infty} \frac{(-1)^{k-1}}{(k-1)\left(\begin{array}{c}
2 k \\
k
\end{array}\right)}=\frac{1}{2}-\frac{3}{2} A_{1}^{-}=\frac{1}{2}-\frac{3}{\sqrt{5}} \ln \phi
\end{aligned}
$$

Again, these equations resemble similar equations for the zeta and eta functions, namely $\sum_{m=2}^{\infty}(\zeta(m)-1)=1$ and $\sum_{m=2}^{\infty}(\eta(m)-1)=1-2 \eta(1)=1-2 \ln 2$.

Finally, the sums $A_{1}^{+}$and $A_{2}^{+}$from (21) and (23), and also $A_{1}^{-}$and $A_{2}^{-}$of (22) and (24), are related by

$$
2 A_{2}^{+}=3\left(A_{1}^{+}\right)^{2}=\frac{\pi^{2}}{9}, \quad 2 A_{2}^{-}=5\left(A_{1}^{-}\right)^{2}=4 \ln ^{2} \phi .
$$

In addition, the relations between $A_{0}^{+}$and $A_{1}^{+}$, and also between $A_{0}^{-}$and $A_{1}^{-}$, are given by (27) and (28):

$$
3 A_{0}^{+}-2 A_{1}^{+}=1, \quad 5 A_{0}^{-}-2 A_{1}^{-}=1 .
$$

Thus, combining (31) and (32), the following double sums are shown to be 0 :

$$
\begin{aligned}
& 0=6 A_{0}^{+} A_{2}^{+}-4 A_{1}^{+} A_{2}^{+}-3 A_{1}^{+} A_{1}^{+}=\sum_{k=1}^{\infty} \sum_{j=1}^{\infty} \frac{6 k^{2}-4 k-3 k j}{k^{2} j^{2}\left(\begin{array}{c}
2 k \\
k
\end{array}\right)\left(\begin{array}{c}
2 j \\
j
\end{array}\right)}, \\
& 0=10 A_{0}^{-} A_{2}^{-}-4 A_{1}^{-} A_{2}^{-}-5 A_{1}^{-} A_{1}^{-}=\sum_{k=1}^{\infty} \sum_{j=1}^{\infty}(-1)^{k+j} \frac{10 k^{2}-4 k-5 k j}{k^{2} j^{2}\left(\begin{array}{c}
2 k \\
k
\end{array}\right)\left(\begin{array}{c}
2 j \\
j
\end{array}\right)} .
\end{aligned}
$$

Here, the question remains open whether these equations could be proved in rational terms, i.e., without an explicit calculation of the irrational sums $A_{1}$ and $A_{2}$.

Acknowledgements I am grateful to Jörn Steuding for his kind support and helpful suggestions. I would also like to thank the referees for a number of valuable improvements and for correcting some errors.

Open Access This article is licensed under a Creative Commons Attribution 4.0 International License, which permits use, sharing, adaptation, distribution and reproduction in any medium or format, as long as you give appropriate credit to the original author(s) and the source, provide a link to the Creative Commons licence, and indicate if changes were made. The images or other third party material in this article are included in the article's Creative Commons licence, unless indicated otherwise in a credit line to the material. If material is not included in the article's Creative Commons licence and your intended use is not permitted by statutory regulation or exceeds the permitted use, you will need to obtain permission directly from the copyright holder. To view a copy of this licence, visit http://creativecommons.org/licenses/by/4.0/. 


\section{References}

1. Apéry, R.: Irrationalité de $\zeta(2)$ et $\zeta(3)$. Astérisque 61, 11-13 (1979)

2. Bailey, D.H., Borwein, J.M., Bradley, D.M.: Experimental determination of Apéry-like identities for $\zeta(2 n+2)$. Experiment. Math. 15(3), 281-289 (2006)

3. Baker, A.: Linear forms in the logarithms of algebraic numbers. I. Mathematika 13, 204-216 (1966)

4. Baker, A.: Linear forms in the logarithms of algebraic numbers. II. Mathematika 14, 102-107 (1967)

5. Baker, A.: Linear forms in the logarithms of algebraic numbers. III. Mathematika 14, 220-228 (1967)

6. Beukers, F.: A note on the irrationality of $\zeta(2)$ and $\zeta(3)$. Bull. London Math. Soc. 11(3), 268-272 (1979)

7. Borwein, J.M., Broadhurst, D.J., Kamnitzer, J.: Central binomial sums, multiple Clausen values and zeta values. Experiment. Math. 10(1), 25-34 (2001)

8. Borwein, J.M., Bradley, D.M.: Empirically determined Apéry-like formulae for $\zeta(4 n+3)$. Experiment. Math. 6(3), 181-194 (1997)

9. Cohen, H.: Démonstration de l'irrationalité de $\zeta$ (3) (d'apres R. Apery). Séminaire de Théorie des Nombres de Grenoble, 6, \# 6 (1978)

10. Comtet, L.: Advanced Combinatorics. Reidel, Dordrecht (1974)

11. Finch, S.R.: Mathematical Constants II. $\$ 1.22$ Central binomial coefficients. Encyclopedia of Mathematics and its Applications, vol. 169, pp. 182-188. Cambridge University Press, Cambridge (2019)

12. Lehmer, D.H.: Interesting series involving the central binomial coefficient. Amer. Math. Monthly 92(7), 449-457 (1985)

13. van der Poorten, A.: A proof that Euler missed. Math. Intell. 1(4), 195-203 (1978)

14. Sprugnoli, R.: Sums of reciprocals of the central binomial coefficients. Integers 6, \# A27 (2006)

15. Taylor, R.: Integrals and interesting series involving the central binomial coefficients (2013). https:// web.williams.edu/Mathematics/sjmiller/public_html/hudson/TaylorR_IntSeries.pdf

Publisher's Note Springer Nature remains neutral with regard to jurisdictional claims in published maps and institutional affiliations. 